\title{
Medication Utilization for Alcohol Use Disorder in a Commercially Insured Population
}

\author{
Haiden A. Huskamp, Ph.D. ' (1), Sharon Reif, Ph.D. ${ }^{2}$, Shelly F. Greenfield, M.D., M.P.H. ${ }^{3,4}$, \\ Sharon-Lise T. Normand, Ph.D. ${ }^{1,5}$, and Alisa B. Busch, M.D., M.S. ${ }^{1,3,4}$
}

\begin{abstract}
'Department of Health Care Policy, Harvard Medical School, 180 Longwood Avenue, Boston, MA, USA; ${ }^{2}$ Heller School for Social Policy and Management, Brandeis University, 415 South Street, Waltham, MA, USA; ${ }^{3}$ McLean Hospital, 115 Mill Street, Belmont, MA, USA; ${ }^{4}$ Department of Psychiatry, Harvard Medical School, Boston, MA, USA; 5 Department of Biostatistics, Harvard T.H. Chan School of Public Health, 677 Huntington Avenue, Boston, MA, USA.
\end{abstract}

\begin{abstract}
OBJECTIVE: Examine patterns of alcohol use disorder (AUD) medication use and identify factors associated with prescription fill among commercially insured individuals with an index AUD visit.
\end{abstract}

DESIGN: Using 2008-2018 claims data from a large national insurer, estimate days to first AUD medication using cause-specific hazards approach to account for competing risk of benefits loss.

PARTICIPANTS: Aged 17-64 with $\geq 1$ AUD visit.

MAIN MEASURE: Days to AUD medication fill.

KEY RESULTS: A total of $13.3 \%$ of the 151,128 with an index visit filled an AUD prescription after that visit, while $69.8 \%$ lost benefits before filling and $17.0 \%$ remained enrolled but did not fill (median days observed $=305$ ). Almost half (46.3\%) of those who filled a prescription received substance use disorder (SUD) inpatient care within 7 days before the fill, and $63.4 \%$ received SUD outpatient care. Likelihood of medication use was higher for those aged $26-35,36-45$, and $46-55$ years relative to $56-$ 64 years (e.g., 26-35: hazard ratio $=1.29$ [95\% confidence interval 1.23-1.36]); those diagnosed with moderate/ severe AUD (2.05 [1.98-2.12]), co-occurring opioid use disorder (OUD) (1.33 [1.26-1.39]), or severe mental illness (1.31 [1.27-1.35]); those with a chronic alcohol-related diagnosis (1.08 [1.04-1.12]); and those whose index visit was in an inpatient/emergency department (1.27 [1.231.31]) or intermediate care setting (1.13 [1.07-1.20]) relative to outpatient. Likelihood of use was higher in later years relative to 2008 (e.g., 2018:2.02 [1.89-2.15]) and higher for those who received the majority of AUD care in a practice with a psychiatrist/addiction medicine specialist (1.13 [1.10-1.16]). Likelihood of use was lower for those diagnosed with a SUD other than AUD or OUD (0.88 [0.85-0.92]), those with an acute alcohol-related condition (0.79 [0.75-0.84]), and males (0.71 [0.69-0.73]).

CONCLUSIONS: While AUD medication use increased and was more common among individuals with greater severity, few patients who could benefit from medications are using them. More efforts are needed to identify and

A set of preliminary results using earlier data was presented at the October 2019 Addiction Health Services Research Conference.

Received January 14, 2020

Accepted July 16, 2020

Published online August 4, 2020 treat individuals in non-acute care settings earlier in their course of AUD.

KEY WORDS: substance use disorder; medication; alcohol use disorder.

J Gen Intern Med 35(11):3262-70

DOI: $10.1007 / \mathrm{s} 11606-020-06073-\mathrm{w}$

(c) Society of General Internal Medicine 2020

A lcohol use disorder (AUD) remains the most common A substance use disorder in the USA. Approximately 14.8 million Americans age 12 or older (or 5.4\%) had $\mathrm{AUD}^{1}$ in 2018, and more than 88,000 die each year from alcohol-related causes. $^{2}$ Guideline-recommended treatments for AUD are available, including psychosocial treatments and medications such as naltrexone, acamprosate, disulfiram, and topiramate. Despite the availability of most of these treatments for two decades or longer, only approximately one in six people with AUD in 2017 received any treatment in the past 12 months. ${ }^{3}$ AUD medications have been particularly slow to diffuse into practice. $^{4-11}$ Barriers to the use of AUD and other substance use disorder (SUD) medications exist, including coverage restrictions such as prior authorization requirements and general shortages of SUD providers. ${ }^{12-17}$ Noting the high societal and personal burdens associated with AUD and the high rate of undertreatment, the American Psychiatric Association released a new practice guideline to inform the prescribing of AUD medications in 2018. ${ }^{18}$

Little is currently known about how AUD medications are being used among commercially insured individuals or factors associated with AUD medication use earlier in the course of illness. One analysis of 2014-2015 Cigna data found that 6.2$7.6 \%$ of enrollees diagnosed with AUD filled an AUD medication prescription each year, although the study did not identify predictors of AUD medication fills. ${ }^{19} \mathrm{We}$ examined factors associated with AUD prescription fill among individuals with an index AUD treatment visit in a large population of commercially insured individuals over an 11-year period from 2008 to 2018. 


\section{METHODS}

Identification of Study Cohort. We used 2008-2018 claims data from a large national commercial insurer. These data are not publicly available under the terms of a data use agreement signed with the insurer. We focused on individuals aged 17 64 who had at least one claim for a face-to-face encounter with an AUD diagnosis (ICD-9 codes = 291.XX, 303.XX, 305.0X; ICD-10 code $=F 10 . X X)$ in the primary or secondary diagnosis field and/or a claim with a CPT code of 99408 or 99409 (codes identifying Screening Brief Intervention and Referral to Treatment (SBIRT) services). We consider the first observed face-to-face AUD claim to be the "index AUD visit."

To be included in the study cohort, an individual must have been continuously enrolled in medical, behavioral health, and pharmacy benefits managed by the insurer for 90 days before (and including) the date of the index AUD visit so we are able to determine that the first observed face-to-face AUD claim does, in fact, represent a new episode, or what we have defined as an index AUD visit. The individual must not have had any claims during that 90-day period for any of four medications used commonly to treat AUD: acamprosate, disulfiram, naltrexone (both oral and injectable formulations), and topiramate. While topiramate, approved by the Food and Drug Administration (FDA) to treat seizure disorders, has not been approved for AUD treatment, it has demonstrated some efficacy in decreasing heavy alcohol use and promoting abstinence among patients with $\mathrm{AUD}^{20,21}$ and is recommended by recent AUD practice guidelines. ${ }^{14,}{ }^{22}$ We used pharmacy claims to determine the use of all medications; for injectable naltrexone, we also used medical claims with a J-code of J2315. During the study period, only injectable naltrexone required prior authorization.

Statistical Analysis. Because the loss of benefits (i.e., due to a change in insurers, becoming uninsured, or death) could impact the likelihood of a future medication fill, we estimated days to first AUD medication use after the index visit using a cause-specific hazards approach to account for the competing risk of loss of medical, behavioral health, and/or pharmacy benefits managed by this insurer. This time-to-event analysis uses all data to follow an individual until no longer enrolled in benefits, rather than setting an arbitrary end-point (e.g., 6 months). We note that some individuals may fill a prescription after losing benefits but this could not be observed. We adopted a cause-specific Cox proportional hazards regression model for the competing risk analysis. Our approach provided estimates of the associations of covariates on the relative change in the instantaneous rate of medication use among subjects who have not lost benefits and who have not yet used a medication. The model assumes that the hazard rates for covariates (e.g., male versus female) are proportional. Proportionality was tested using a Kolmogorov- type test and influential observations were identified using the deviance residual (see Appendix).

Key Variables. The model included age category (17-25, 26$35,36-45,46-55,56$ and older), male, employee status (employee vs. dependent), residence in a rural area, region (Northeast, Midwest, West, South), median household income for zip code of residence (in quartiles), and indicator variables for the year of the index visit. We also included the following patient clinical characteristics at baseline (i.e., the 90 days before and including the index visit date): diagnosis of moderate/severe AUD, opioid use disorder (OUD), SUD other than AUD or OUD, severe mental illness (schizophrenia and psychotic disorders, bipolar disorder, or major depression), acute alcohol-related condition (e.g., alcoholic gastritis, acute pancreatitis, alcoholic psychosis), and chronic alcohol-related condition (see Appendix for the list of diagnosis codes used). ${ }^{23}$ Because our cohort consists only of individuals who have been diagnosed with AUD, we included in the definitions of acute and chronic alcohol-related variables diagnoses that have direct attributable fractions related to AUD, even if < $100 \%$ attributable, since conditions less than $100 \%$ attributable to alcohol use would still likely be exacerbated by it and would lead to poorer health outcomes and greater alcoholattributed health utilization (e.g., esophageal varices, seizure disorder). In addition, we included a variable indicating whether the practice (identified using a Taxpayer Identification Number or TIN) in which the patients received the majority of their AUD care had a psychiatrist or addiction medicine specialist billing the insurer during the year of the index visit. We also included two variables indicating whether the index visit occurred in an inpatient/emergency department setting or an intermediate care setting (i.e., partial hospitalization, residential, intensive outpatient), relative to an outpatient setting.

Descriptive Analyses of Medications and SUD Services Used by AUD Medication Users. For those who filled an AUD medication prescription after their index visit, we identified the first medication used. To examine medication continuation, we also calculated the percentage of days with AUD medication supplied during the first 180 days after (and including) the first fill date among individuals who filled an AUD medication prescription. In addition, we examined the use of specific types of SUD services (inpatient, residential, partial hospitalization, intensive outpatient, emergency department, and outpatient; detoxification services are included by setting) within the 7 days before (and including) the first AUD medication fill to determine the types of care used that might be associated with the first fill. To consider a claim to be for a SUD service, the primary diagnosis had to be SUD (ICD-9 291-292, 303, 304, 305.0, 305.2-305.7, 305.9; ICD-10: F10-F16, F18- 
F19). Emergency department visits that resulted in hospitalization were counted as inpatient and not emergency department care.

\section{RESULTS}

We identified 154,443 person-years with an index AUD visit from among individuals age 17-64 who met the enrollment criteria described above (ranging from 8.2 million to 9.6 million people, depending on the year). We dropped 3315 with missing zip code and/or gender information. Although there were statistically significant differences across those missing this information and those who were not in selected characteristics (e.g., age, diagnosis of moderate or severe AUD), these differences were generally small in magnitude (see Appendix). The final cohort included 151,128 individuals. Median observation time after the index visit for individuals in the cohort was 305 days.

Table 1 provides descriptive information on the final cohort. A psychiatrist or addiction medicine specialist billed in the practice where the majority of AUD care was received for $40.4 \%$ of individuals. Over one-third (38.4\%) of index visits occurred in an inpatient/emergency department setting, whereas $6.1 \%$ occurred in an intermediate care setting, and $55.6 \%$ in an outpatient setting.

Patterns of AUD Medication Use. Over the 11-year study period, approximately $13.3 \%(n=20,058)$ filled an AUD medication prescription after their index visit (ranging from a low of $10.7 \%$ in 2008 to a high of $15.4 \%$ in 2016, Fig. 1), while $69.8 \%$ lost benefits before filling a prescription and $17.0 \%$ remained enrolled through the end of the study period but did not fill a prescription. For those who filled a prescription before losing benefits, the median number of days until fill was 33, with an interquartile range (IQR) of 7209. For those who lost benefits before filling a prescription, the median number of days from index visit until benefits loss was 324 (IQR, 135, 684). Among individuals who did not fill a prescription and maintained all benefits through the end of 2018, median days from index visit to study period end was 556 (IQR, 215, 1217).

Of the 20,058 who filled a prescription before losing benefits, $41.9 \%(n=8407)$ used oral naltrexone for their first fill, $21.9 \%(n=4384)$ acamprosate, $13.2 \%(n=2646)$ topiramate, $16.6 \%(n=3321)$ disulfiram, and 6.5\% $(n=1300)$ injectable naltrexone (Table 2). For these 20,058 (as opposed to the full cohort described above), the index visit occurred in an inpatient setting or emergency department for $46.1 \%$, in an intermediate care setting for $7.1 \%$, and in an outpatient setting for $46.8 \%$ (data not shown). Among individuals using injectable naltrexone, also indicated for OUD, 37.0\% had a baseline OUD diagnosis. While topiramate might have been used to treat seizure disorders rather than AUD in this population, only
Table 1 Descriptive Characteristics of Commercially Insured Individuals Ages 17-64 with Index Alcohol Use Disorder (AUD) Visit $(n=151,128)$

\begin{tabular}{|c|c|}
\hline & $N(\%)$ \\
\hline \multicolumn{2}{|l|}{ Age } \\
\hline $17-25$ & 26,720 \\
\hline $26-35$ & $(17.7)$ \\
\hline $36-45$ & 28,590 \\
\hline $46-55$ & $(18.9)$ \\
\hline \multirow[t]{5}{*}{56 and over } & $\begin{array}{l}34,431 \\
(228)\end{array}$ \\
\hline & 38,858 \\
\hline & $(25.7)$ \\
\hline & 22,529 \\
\hline & $(14.9)$ \\
\hline Male & $\begin{array}{l}100,253 \\
(66.3)\end{array}$ \\
\hline \multicolumn{2}{|l|}{ Employee status } \\
\hline Employee & 96,413 \\
\hline \multirow[t]{2}{*}{ Dependent } & $(63.8)$ \\
\hline & $\begin{array}{l}54,715 \\
(36,2)\end{array}$ \\
\hline Rural & $10.603(70)$ \\
\hline \multicolumn{2}{|l|}{ Region } \\
\hline Northeast & 46,749 \\
\hline Midwest & $(30.9)$ \\
\hline West & 22,390 \\
\hline \multirow[t]{4}{*}{ South } & $(14.8)$ \\
\hline & 32,300 \\
\hline & $\begin{array}{l}(21.4) \\
49.69\end{array}$ \\
\hline & $\begin{array}{l}49,689 \\
(329)\end{array}$ \\
\hline Psychiatrist or addiction medicine specialist billing in & 60,996 \\
\hline practice where patient received most AUD care & $(40.4)$ \\
\hline Moderate or severe AUD diagnosis at baseline & $\begin{array}{l}93,230 \\
(61.7)\end{array}$ \\
\hline OUD diagnosis at baseline & $10,578(7.0)$ \\
\hline At least 1 substance use disorder (SUD) diagnosis & 27,652 \\
\hline besides AUD or OUD at baseline & $(18.3)$ \\
\hline Hallucinogens & $8835(5.7)$ \\
\hline Cannabis & $14,409(9.3)$ \\
\hline Sedatives & $4749(3.1)$ \\
\hline Cocaine & $6883(4.5)$ \\
\hline Amphetamines & $3096(2.0)$ \\
\hline Acute alcohol-related medical diagnosis at baseline & $12,666(8.2)$ \\
\hline Chronic alcohol-related medical diagnosis at baseline & $\begin{array}{l}23,176 \\
(15.0)\end{array}$ \\
\hline Severe mental illness diagnosis at baseline & $\begin{array}{l}32,395 \\
(21.4)\end{array}$ \\
\hline \multirow{2}{*}{$\begin{array}{l}\text { Psychiatrist or addiction medicine specialist claim at } \\
\text { baseline } \\
\text { Any inpatient SUD use at baseline }\end{array}$} & $\begin{array}{l}28.785 \\
(19.1)\end{array}$ \\
\hline & 63,074 \\
\hline \multicolumn{2}{|l|}{ Site of index AUD visit } \\
\hline \multirow{2}{*}{$\begin{array}{l}\text { Inpatient or emergency department } \\
\text { Intermediate care }\end{array}$} & 59,289 \\
\hline & $(38.4)$ \\
\hline \multirow[t]{2}{*}{ Outpatient } & $9346(6.1)$ \\
\hline & $\begin{array}{l}85,808 \\
(55.6)\end{array}$ \\
\hline \multicolumn{2}{|l|}{ Year of index AUD visit } \\
\hline 2018 & $14,032(9.3)$ \\
\hline 2017 & $14,337(9.5)$ \\
\hline 2016 & $13,986(9.3)$ \\
\hline 2015 & $12,908(8.5)$ \\
\hline 2014 & $12,361(8.2)$ \\
\hline 2013 & $11,414(7.6)$ \\
\hline 2012 & $11,781(7.8)$ \\
\hline 2011 & $12,372(8.2)$ \\
\hline 2010 & $13,687(9.1)$ \\
\hline 2009 & 16,403 \\
\hline \multirow{3}{*}{2008} & (10.9) \\
\hline & 17,847 \\
\hline & (11.8) \\
\hline
\end{tabular}

Notes: The baseline period was 90 days before and including the index AUD visit 


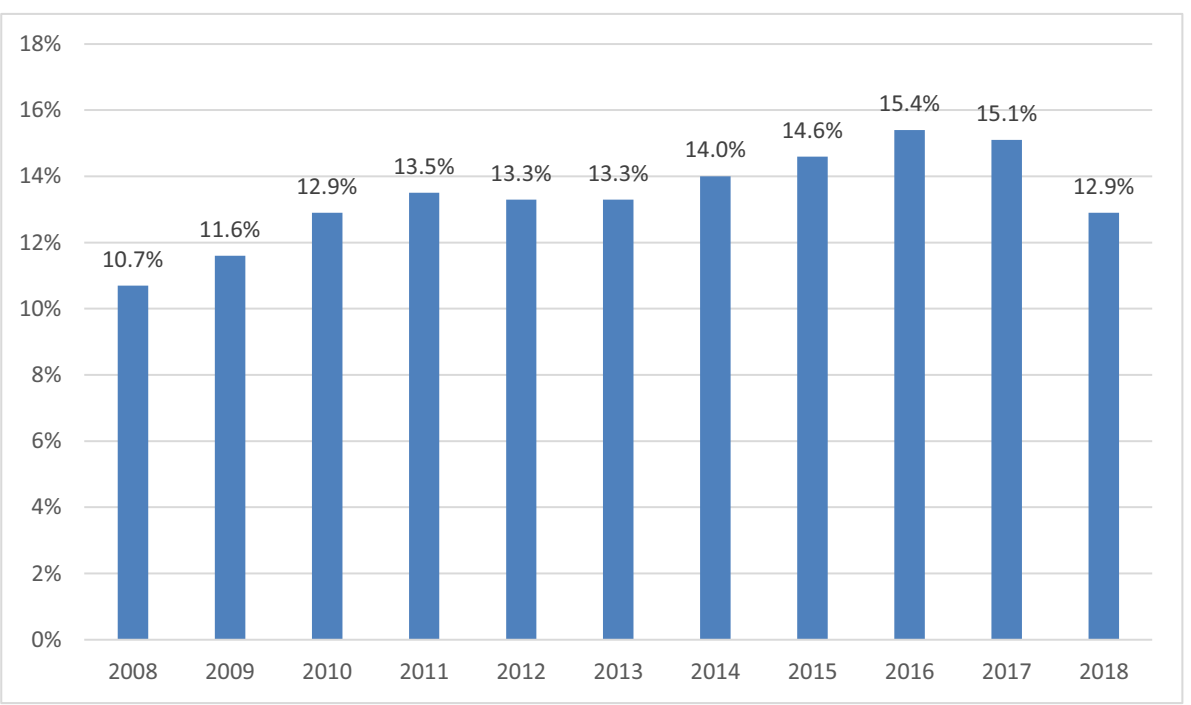

Figure 1 Percent of individuals with an index AUD visit who fill an AUD medication prescription while maintaining insurance benefits, 20082018.

$13.1 \%$ of the topiramate users had a baseline diagnosis of seizure disorder (data not shown). The median percentage of days with AUD medication supplied during the first 180 days after (and including) the first fill date was 33\% (interquartile range, $17 \%, 58 \%)$.

Almost half (46.3\%) of medication users received SUD inpatient care in the 7 days before (and including) the day they filled their first AUD prescription after the index visit (Table 3). Approximately $4.0 \%$ used residential care, 3.1\% partial hospital care, $5.3 \%$ intensive outpatient care, $4.1 \%$ emergency department care for SUD, and $63.4 \%$ SUD outpatient care during this 7-day period.

Factors Associated with AUD Medication Use. In models that account for the competing risk of benefit loss, likelihood of medication use was lower for males than females (hazard ratio $[95 \% \mathrm{CI}]=0.71[0.69-0.73]$; those age $17-25$ relative to those 56-64 (0.76 [0.71-0.80]); employees relative to dependents (0.89 [0.86-0.91]); and those residing in the Northeast, Midwest, and West, all relative to the South (e.g., Northeast: 0.79 [0.76-0.82]) (Table 4). It was also lower for individuals diagnosed at baseline with a co-occurring SUD other than AUD or OUD (0.88 [0.85-0.92]) and for those with an acute alcohol-related condition (0.79 [0.75-0.84]). Likelihood of AUD medication use was higher for those 26-35, 36-45, and 46-55 relative to those 56-64 (e.g., 26-35: 1.29 [1.231.36]) and for those with moderate/severe AUD (2.05 [1.98$2.12])$, co-occurring opioid use disorder (1.33 [1.26-1.39]), or severe mental illness (1.31 [1.27-1.35]). Those diagnosed with a chronic alcohol-related diagnosis had higher likelihood of medication use than those without such a diagnosis (1.08 [1.04-1.12]), and individuals whose index visit was either in an inpatient/emergency department setting (1.27 [1.23-1.31]) or an intermediate care setting (1.13 [1.07-1.20]) had a higher likelihood relative to those whose visit occurred in an outpatient setting. Having a psychiatrist/addiction specialist billing in the practice had a positive association with the likelihood of medication use (1.13 [1.10-1.16]). Likelihood was higher for individuals living in higher income quartiles (e.g., median income quartile 4 vs. 1: 1.51 [1.45-1.58]) and higher in later years relative to 2008 (e.g., 2018: 2.02 [1.892.15]). (See Appendix for results for factors associated with likelihood of losing benefits before filling an AUD medication prescription and modeling assumptions.)

\section{DISCUSSION}

In a large, national population of commercially insured individuals, a relatively small percentage of those with an index AUD visit-13.3\%-filled a prescription for an AUD medication after that visit, while maintaining insurance benefits with this insurer. Among medication users, the median number of days between index visit and first fill was 33, or just over a month. Loss of benefits is unlikely to be a key reason for not filling a prescription soon after an index visit since the median number of days before loss of benefits without filling a prescription is 324. Interestingly, a large proportion of AUD

Table 2 Alcohol Use Disorder (AUD) Medications Used for First Prescription Fill after the Index AUD Visit $(N=\mathbf{2 0 , 0 5 8})$

\begin{tabular}{ll}
\hline \hline & No. (\%) \\
\hline Oral naltrexone & $8407(41.9)$ \\
Acamprosate & $4384(21.9)$ \\
Topiramate & $2646(13.2)$ \\
Disulfiram & $3321(16.6)$ \\
Injectable naltrexone & $1300(6.5)$ \\
\hline
\end{tabular}

Notes: The denominator includes those who filled a prescription for an $A U D$ medication after the index AUD visit and while maintaining insurance benefits with the large national insurer studied 
Table 3 Substance Use Disorder (SUD) Service Use in the 7 Days before and including the First Alcohol Use Disorder (AUD) Medication Fill among Those with Index AUD Visit who Fill an AUD Medication During the Study Period $(n=20,058)$

\begin{tabular}{ll}
\hline \hline & No. (\%) \\
\hline SUD inpatient care & $9292(46.3)$ \\
SUD residential care & $803(4.0)$ \\
SUD partial hospital care & $618(3.1)$ \\
SUD intensive outpatient care & $1072(5.3)$ \\
SUD emergency department care & $830(4.1)$ \\
SUD outpatient care & $12,714(63.4)$ \\
\hline
\end{tabular}

medication users were using intensive SUD services in the week before they filled their first prescription. For example, 46.3\% used SUD inpatient care during this period. This finding suggests that medication users may have been sicker on average and/or perhaps more engaged in intensive SUD treatment relative to non-medication users. This is also consistent with our finding that moderate/severe AUD (compared to mild) and chronic alcohol-related medical conditions were associated with a higher likelihood of receiving medication.

It is notable that over a third of index visits in this population $(38.4 \%)$ occurred in inpatient/emergency department settings and not in outpatient settings and that only approximately one in five $(19.1 \%)$ individuals in the cohort saw a psychiatrist or addiction medicine specialist in the 90 days before and including the index visit. Prior research has documented the prevalence of AUD in primary care populations. ${ }^{24}$ The US Preventive Services Task Force recommends screening and brief intervention treatment (SBIRT) in primary care settings due to its efficacy in identifying alcohol use among those at risk of AUD. ${ }^{25,}{ }^{26}$ Our finding that many index visits are occurring in inpatient or emergency department settings highlights the important public health opportunity in identifying individuals in non-urgent care settings and, ideally, earlier in the course of their AUD. Although most (59.6\%) people with an index visit were primarily treated for AUD in practices with no psychiatrist or addiction medicine specialist, patients in practices with these specialists had a higher likelihood of medication use potentially because of enhanced access to consultation and referral as needed. Improving training and specialty consultations to primary care physicians in the treatment of AUD such as through the Extension for Community Healthcare Outcomes $(\mathrm{ECHO})^{27}$ and other telehealth models of consultation and training may enhance primary care physicians' confidence in their ability to treat this population. Collaborative care models of clinical integration of behavioral health care and primary care may also help improve access to AUD treatment including medications.

We found that while approximately two-thirds $(66.3 \%)$ of individuals with a face-to-face visit for AUD in a given calendar year were men, men were less likely to than women to fill a prescription for an AUD medication while maintaining their insurance benefits. This difference was not explained by
Table 4 Results from Competing Risk Model of Alcohol Use Disorder (AUD) Medication Use after Index AUD Visit, Accounting for Risks of Benefit Loss

\begin{tabular}{|c|c|c|}
\hline & Hazard ratio & $95 \%$ confidence interval \\
\hline \multicolumn{3}{|l|}{ Age } \\
\hline $17-25$ & 0.76 & $0.71,0.80$ \\
\hline $26-35$ & 1.29 & $1.23,1.36$ \\
\hline $36-45$ & 1.47 & $1.40,1.54$ \\
\hline $46-55$ & 1.31 & $1.25,1.38$ \\
\hline 56 and over & Reference & - \\
\hline \multicolumn{3}{|l|}{ Male } \\
\hline Yes & 0.71 & $0.69,0.73$ \\
\hline No & Reference & - \\
\hline \multicolumn{3}{|c|}{ Employee status } \\
\hline Employee & 0.89 & $0.86,0.91$ \\
\hline Dependent & Reference & - \\
\hline \multicolumn{3}{|l|}{ Rural } \\
\hline Yes & 0.95 & $0.90,1.01$ \\
\hline \multirow{2}{*}{\multicolumn{3}{|c|}{ Region }} \\
\hline & & \\
\hline Northeast & 0.79 & $0.76,0.82$ \\
\hline Midwest & 0.88 & $0.85,0.92$ \\
\hline West & 0.93 & $0.89,0.96$ \\
\hline South & Reference & - \\
\hline \multicolumn{3}{|c|}{ Median household income for zip code of residence, in quartiles } \\
\hline Quartile 1 & Reference & - \\
\hline Quartile 2 & 1.17 & $1.12,1.22$ \\
\hline Quartile 3 & 1.29 & $1.24,1.35$ \\
\hline Quartile 4 & 1.51 & $1.45,1.58$ \\
\hline \multicolumn{3}{|c|}{$\begin{array}{l}\text { Psychiatrist or addiction medicine specialist billing in practice where } \\
\text { patient received most AUD care }\end{array}$} \\
\hline Yes & 1.13 & $1.10,1.16$ \\
\hline No & Reference & - \\
\hline \multicolumn{3}{|c|}{ Moderate or severe AUD diagnosis at baseline } \\
\hline Yes & 2.05 & $1.98,2.12$ \\
\hline No & Reference & - \\
\hline \multicolumn{3}{|c|}{ OUD diagnosis at baseline } \\
\hline Yes & 1.33 & $1.26,1.39$ \\
\hline No & Reference & - \\
\hline \multicolumn{3}{|c|}{ SUD diagnosis besides AUD or OUD at baseline } \\
\hline Yes & 0.88 & $0.85,0.92$ \\
\hline No & Reference & - \\
\hline \multicolumn{3}{|c|}{ Acute alcohol-related medical diagnosis at baseline } \\
\hline Yes & 0.79 & $0.75,0.84$ \\
\hline No & Reference & - \\
\hline \multicolumn{3}{|c|}{ Chronic alcohol-related medical diagnosis at baseline } \\
\hline Yes & 1.08 & $1.04,1.12$ \\
\hline No & Reference & - \\
\hline \multicolumn{3}{|c|}{ Severe Mental Illness Diagnosis at Baseline } \\
\hline Yes & 1.31 & $1.27,1.35$ \\
\hline No & Reference & - \\
\hline \multicolumn{3}{|c|}{ Index AUD visit setting } \\
\hline Inpatient/ED & 1.27 & $1.23,1.31$ \\
\hline Intermediate & 1.13 & $1.07,1.20$ \\
\hline Outpatient & Reference & - \\
\hline \multicolumn{3}{|c|}{ Year of index AUD visit } \\
\hline 2018 & 2.02 & $1.89,2.15$ \\
\hline 2017 & 1.81 & $1.70,1.93$ \\
\hline 2016 & 1.71 & $1.61,1.82$ \\
\hline 2015 & 1.59 & $1.49,1.69$ \\
\hline 2014 & 1.50 & $1.40,1.60$ \\
\hline 2013 & 1.36 & $1.27,1.46$ \\
\hline 2012 & 1.29 & $1.21,1.38$ \\
\hline 2011 & 1.28 & $1.20,1.37$ \\
\hline 2010 & 1.24 & $1.16,1.32$ \\
\hline 2009 & 1.12 & $1.05,1.19$ \\
\hline 2008 & Reference & - \\
\hline
\end{tabular}

Notes: We estimated the risk of AUD medication use controlling for patient characteristics and whether the practice where the patient received the majority of AUD care included a psychiatrist or addiction medicine specialist, accounting for the competing risk of loss of benefits. The baseline period was 90 days before and including the index AUD visit

insurance status as there was also no significant gender difference in the likelihood of losing benefits before filling a 
prescription for an AUD medication (Appendix Table 6). Previous research has demonstrated that women are less likely to receive treatment for AUD through the lifespan than men, but once in treatment there are few gender differences reported in clinical outcomes including medication treatment with naltrexone and acamprosate. ${ }^{28,}{ }^{29}$ To our knowledge, this is the first study to report a gender difference in filling a prescription for an AUD medication following a face-to-face visit with women being more likely to do so. It is unclear whether in this population physicians were more likely to prescribe medication for women than men, or women were more likely to adhere to the recommendation and fill the prescription than men. More research is needed to understand gender differences in the acceptability of various treatments for AUDs including medications.

Also notable is the finding that a diagnosis of an acute alcohol-related medical condition was negatively associated with likelihood of medication use, whereas a diagnosis of a chronic alcohol-related medical condition was positively associated with it. We also found that those with a baseline diagnosis of an acute alcohol-related medical condition had a higher rate of moderate or severe AUD at baseline than did individuals without an acute alcohol-related medical condition diagnosis $(70.9 \%$ vs. $60.9 \%$, respectively; $p<0.0001$ ). Thus, low rates of more severe AUD do not explain this finding among those with acute alcohol-related conditions. Future research is needed to better understand this finding; nonetheless, it is worrisome that these individuals were less likely to receive AUD medications.

There are several limitations of our analysis. First, individuals identified as having AUD and receiving treatment may be undercounted. Due to concerns about stigma and concerns regarding employer knowledge about their AUD, some patients may not disclose alcohol use, providers may not code AUD diagnoses in claims, and some patients may choose to pay out-of-pocket rather than bill their insurance. However, we were examining medications among individuals for whom an AUD diagnosis was already present in the claims. Also, some enrollees may have received AUD treatment through an employee assistance program or at public, state-funded SUD programs that did not bill insurance. In addition, we may be overestimating days to medication use in some cases because we are unable to observe AUD medications dispensed by inpatient or intermediate care settings under bundled payment rates (for example, during an inpatient stay or when an individual is discharged with a limited supply of medication for use in the community). Finally, we studied a single large national insurer; consequently, our findings may not be generalizable to enrollees in other commercial plans. Nevertheless, this large insurer enrolls individuals in all 50 states and the District of Columbia, with approximately 20 million members per year.

\section{CONCLUSIONS}

Multiple meta-analyses have found that AUD medications are efficacious and offer clinical benefit for patients with AUD. ${ }^{30}$, ${ }^{31}$ Our results suggest that AUD medication use is more common among those with more severe disease or illness complexity (e.g., moderate/severe AUD, co-occurring OUD or severe mental illness or co-occurring chronic medical conditions often associated with AUD). While this finding suggests that patients who are the most ill or complicated are more likely to receive medications, it is nonetheless concerning that more patients who could benefit from them are not currently filling prescriptions. Much work is needed to increase the dissemination of effective pharmacotherapies into routine care for patients with AUD.

Acknowledgments: Hocine Azeni, M.A., provided expert statistical programming.

Corresponding Author: Haiden A. Huskamp, Ph.D.; Department of Health Care Policy, Harvard Medical School, 180 Longwood Avenue, Boston, MA 02115, USA (e-mail: huskamp@hcp.med.harvard.edu).

Funding Information This work was funded by the National Institute on Drug Abuse (P3O DA035772 Brandeis/Harvard NIDA Center to Improve System Performance of Substance Use Disorder Treatment).

\section{Compliance with Ethical Standards:}

Conflict of Interest: The authors declare that they do not have a conflict of interest.

\section{REFERENCES}

1. Substance Abuse and Mental Health Services Administration. Key Substance Use and Mental Health Indicators in the United States: Results from the 2018 National Survey on Drug Use and Health. HHS Publication No. PEP19-5068, NSDUH Series H-54. Rockville, MD: Center for Behavioral Health Statistics and Quality, Substance Abuse and Mental Health Services Administration. Available at: https://www. samhsa.gov/data/. Accessed January 13, 2020.

2. Centers for Disease Control and Prevention. Alcohol and Public Health: Alcohol-Related Disease Impact (ARDI) - Average for United States 2006 2010 Alcohol-Attributable Deaths Due to Excessive Alcohol Use. Available at: www.cdc.gov/ARDI. Accessed September 23, 2019.

3. Substance Abuse and Mental Health Services Administration. Results from the 2017 National Survey on Drug Use and Health: Detailed Tables. Available at: https://www.samhsa.gov/data/sites/default/files/cbhsqreports/NSDUHDetailedTabs2017/NSDUHDetailedTabs2017.htm\#tab512A. Accessed January 13, 2020.

4. Knudsen HK, Abraham AJ, Roman PM. Adoption and implementation of medications in addiction treatment programs. J Addict Med. 2011;5(1):21-27

5. Iheanacho T, Issa M, Marienfeld C, Rosenheck $\mathbf{R}$. Use of naltrexone for alcohol use disorders in the Veterans' Health Administration: a national study. Drug Alcohol Depend. 2013;132(1-2):122-126

6. Mark TL, Kassed CA, Vandivort-Warren R, Levit KR, Kranzler HR. Alcohol and opioid dependence medications: prescription trends, overall and by physician specialty. Drug Alcohol Depend. 2009;99(1-3):345-349.

7. Rieckmann T, Muench J, McBurnie MA, et al. Medication-assisted treatment for substance use disorders within a national community health center research network. Subst Abus. 2016;37(4):625-634

8. Harris AH, Oliva E, Bowe T, Humphreys KN, Kivlahan DR, Trafton JA Pharmacotherapy of alcohol use disorders by the Veterans Health Administration: patterns of receipt and persistence. Psychiatr Serv. 2012;63(7):679-685 
9. Knudsen HK, Roman PM. Service delivery and pharmacotherapy for alcohol use disorder in the era of health reform: Data from a national sample of treatment organizations. Subst Abus. 2016;37(1):230-237

10. Harris AH, Kivlahan DR, Bowe T, Humphreys KN. Pharmacotherapy of alcohol use disorders in the Veterans Health Administration. Psychiatr Serv. 2010; 61(4): 392-8

11. Mark TL, Kranzler HR, Song X, Bransberger P, Poole VH, Crosse S. Physician's opinions about medications to treat alcoholism. Addiction. 2003; 98(5): 617-26.

12. Huskamp HA, Riedel LE, Barry CL, Busch AB. Coverage of medications that treat opioid use disorder and opioids for pain management in Marketplace plans. Med Care. 2018;56(6):505-509.

13. Reif S, Horgan CM, Hodgkin D, Matteucci AM, Creedon TB, Stewart MT. Access to Addiction Pharmacotherapy in Private Health Plans. J Subst Abuse Treat. 2016;66:23-29.

14. Horgan CM, Reif S, Hodgkin D, Garnick DW, Merrick EL. Availability of addiction medications in private health plans. J Subst Abuse Treat. 2008;34(2):147-156.

15. Harris AHS, Ellerbe L, Reeder RN, et al. Pharmacotherapy for alcohol dependence: perceived treatment barriers and action strategies among Veterans Health Administration service providers. Psychol Serv. 2013; 10(4): 410-419.

16. Abraham AJ, Ducharme LJ, Roman PM. Counselor attitudes toward pharmacotherapies for alcohol dependence. J Stud Alcohol Drugs. 2009; 70(4): 628-35

17. Hoge MA, Stuart GW, Morris J, Flaherty MT, Paris M, Jr., Goplerud E. Mental health and addiction workforce development: federal leadership is needed to address the growing crisis. Health Aff (Millwood). 2013;32(11):2005-2012.

18. The American Psychiatric Association. Practice Guideline for the Pharmacological Treatment of Patients with Alcohol Use Disorder. Available at: https://psychiatryonline.org/doi/pdf/10.1176/appi.books. 9781615371969. Accessed January 13, 2020.

19. Thomas CP, Ritter GA, Harris AHS, Garnick DW, Freedman KI Herbert B. Applying American Society of Addiction Medicine Performance Measures in Commercial Health Insurance and Services Data. J Addict Med. 2018;12(4):287-294.

20. Paparrigopoulos T, Tzavellas E, Karaiskos D, Kourlaba G, Liappas I. Treatment of alcohol dependence with low-dose topiramate: an open-label controlled study. BMC Psychiatry. 2011;11:41

21. Johnson BA, Rosenthal N, Capece JA, et al. Topiramate for treating alcohol dependence: a randomized controlled trial. JAMA. 2007;298(14):1641-1651.

22. Department of Veterans Affairs, Department of Defense. VA/DoD Clinical Practice Guideline for the Management of Substance Use Disorders. Version 3.0. Available at: https://www.healthquality.va.gov/guidelines/ $\mathrm{MH}$ /sud/VADoDSUDCPGRevised22216.pdf. Accessed January 13, 2020.

23. Centers for Disease Control and Prevention. Alcohol and Public Health: Alcohol-Related Disease Impact (ARDI): Alcohol-Related ICD10 Codes. Available at: https://nccd.cdc.gov/DPH_ARDI/Info/ICDCodes.aspx. Accessed September 23, 2019.

24. Kaner EF, Beyer FR, Muirhead C, et al. Effectiveness of brief alcohol interventions in primary care populations. Cochrane Database Syst Rev. 2018;2:CD004148.

25. O'Connor EA, Perdue LA, Senger CA, et al. Screening and Behaviora Counseling Interventions to Reduce Unhealthy Alcohol Use in Adolescents and Adults: Updated Evidence Report and Systematic Review for the US Preventive Services Task Force. JAMA. 2018;320(18):1910-1928.

26. Curry SJ, Krist AH, Owens DK, et al. Screening and Behavioral Counseling Interventions to Reduce Unhealthy Alcohol Use in Adolescents and Adults: US Preventive Services Task Force Recommendation Statement. JAMA. 2018;320(18):1899-1909.

27. University of New Mexico School of Medicine. About Echo. Available at: https://echo.unm.edu/about-echo. Accessed September 23, 2019.

28. Greenfield SF, Pettinati HM, O'Malley S, Randall PK, Randall CL. Gender differences in alcohol treatment: an analysis of outcome from the COMBINE study. Alcohol Clin Exp Res. 2010;34(10):1803-12.

29. Newberry S, Booth M, Rutter CM, et al. Gender Differences in Response to Alcohol Use Disorder Treatment: A Systematic Review. Santa Monica, CA: RAND Corporation, 2019. Available at: https://www.rand.org/pubs/ research_reports/RR2178.html. Accessed May 12, 2020.

30. Maisel NC, Blodgett JC, Wilbourne PL, Humphreys K, Finney JW. Meta-analysis of naltrexone and acamprosate for treating alcohol use disorders: when are these medications most helpful? Addiction. 2013;108(2):275-293.
31. Rosner S, Leucht S, Lehert P, Soyka M. Acamprosate supports abstinence, naltrexone prevents excessive drinking: evidence from a meta-analysis with unreported outcomes. J Psychopharmacol. 2008;22(1):11-23.

Publisher's note Springer Nature remains neutral with regard to jurisdictional claims in published maps and institutional affiliations.

\section{APPENDIX \\ Defining Moderate/Severe AUD}

We defined moderate/severe AUD using the following ICD9 and ICD10 codes: ICD9: 291.0-291.3, 291.5, 291.8, 291.9303.0, 303.9; ICD10: F10.2x. Therefore, individuals who did not have a "severe AUD" diagnosis were those having the "residual" ICD9/ICD10 codes (305.0x, F10.1x) and the SBIRT codes.

\section{Defining Acute and Chronic Alcohol-related Medical Conditions}

The variable indicating a diagnosis of an acute or chronic alcohol-related condition during the baseline period equals 1 if the individuals had a diagnosis of one of the following during the 90-day baseline period (Appendix Table 5). The diagnoses were inclusive of the Centers for Disease Control and Prevention (CDC) Alcohol Related ICD Codes for acute and chronic conditions that were either $100 \%$ attributable to alcohol use disorders or had an attributable fraction (Centers for Disease Control and Prevention. Alcohol and Public Health: Alcohol-Related Disease Impact (ARDI): Alcohol-Related ICD10 Codes. https://nccd.cdc. gov/DPH_ARDI/Info/ICDCodes.aspx. Accessed September 23,

Table 5 Diagnosis Codes Used to Identify Acute and Chronic Alcohol-related Conditions

\begin{tabular}{lll}
\hline \hline & $\begin{array}{l}\text { ICD-9 } \\
\text { code }\end{array}$ & ICD-10 code \\
& & \\
\hline Acute alcohol conditions & 535.3 & $\mathrm{~K} 29.2$ \\
Alcoholic gastritis & 577.0 & $\mathrm{~K} 85$ \\
Acute pancreatitis & 291 & $\mathrm{~F} 10.3-\mathrm{F} 10.9$ \\
Alcoholic psychosis & & \\
Chronic alcohol conditions & 357.5 & $\mathrm{G} 62.1$ \\
Alcohol polyneuropathy & $*$ & $\mathrm{G} 31.2$ \\
Degeneration of nervous system & & \\
due to alcohol & & $\mathrm{G} 72.1$ \\
Alcoholic myopathy & 425.5 & $\mathrm{I} 42.6$ \\
Alcoholic cardiomyopathy & $571.0-$ & $\mathrm{K} 70-\mathrm{K} 70.4, \mathrm{~K} 70.9$ \\
Alcoholic liver disease & 571.3 & \\
& $*$ & $\mathrm{~K} 86.0$ \\
Alcohol chronic induced & & \\
pancreatitis & 577.1 & $\mathrm{~K} 86.1$ \\
Chronic pancreatitis & 345 & $\mathrm{G} 40, \mathrm{G} 41$ \\
Seizure disorder & $456.0-$ & $\mathrm{I} 85, \mathrm{I} 98.2$ \\
Esophageal varices & 456.2 & \\
Gastroesophageal hemorrhage & 530.7 & $\mathrm{~K} 22.6$ \\
Liver cirrhosis unspecified & $571.5-$ & $\mathrm{K} 74.3-\mathrm{K} 74.6$, \\
& 571.9 & $\mathrm{~K} 76.0, \mathrm{~K} 76.9$ \\
Portal hypertension & 572.3 & $\mathrm{~K} 76.6$ \\
Spontaneous abortion & 634 & $\mathrm{O} 03$ \\
Korsakoff's dementia & 291.1 & $\mathrm{~F} 10.26$ \\
\hline
\end{tabular}

*No ICD9 code and condition is new to ICD10 
Table 6 Results from Competing Risk Model of Risk of Losing Benefits after AUD Index Visit, Accounting for Risks of AUD Medication Use

\begin{tabular}{|c|c|c|}
\hline & Hazard ratio & $95 \%$ confidence interval \\
\hline \multicolumn{3}{|l|}{ Age } \\
\hline $17-25$ & 1.20 & $1.17,1.23$ \\
\hline $26-35$ & 1.36 & $1.33,1.39$ \\
\hline $36-45$ & 1.14 & $1.11,1.16$ \\
\hline $46-55$ & 1.03 & $1.01,1.05$ \\
\hline \multirow{2}{*}{\multicolumn{3}{|c|}{ Male }} \\
\hline & & \\
\hline Yes & 0.99 & $0.98,1.00$ \\
\hline No & Reference & - \\
\hline \multicolumn{3}{|c|}{ Employee status } \\
\hline Employee & 1.16 & $1.15,1.18$ \\
\hline Dependent & Reference & - \\
\hline \multicolumn{3}{|l|}{ Rural } \\
\hline Yes & 0.96 & $0.93,0.98$ \\
\hline No & Reference & - \\
\hline \multicolumn{3}{|l|}{ Region } \\
\hline Northeast & 1.02 & $1.00,1.03$ \\
\hline Midwest & 1.05 & $1.03,1.07$ \\
\hline West & 1.03 & $1.01,1.05$ \\
\hline South & Reference & - \\
\hline \multicolumn{3}{|c|}{ Median household income for zip code of residence } \\
\hline Quartile 1 & Reference & - \\
\hline Quartile 2 & 0.94 & $0.92,0.96$ \\
\hline Quartile 3 & 0.92 & $0.91,0.94$ \\
\hline Quartile 4 & 0.89 & $0.87,0.90$ \\
\hline \multicolumn{3}{|c|}{$\begin{array}{l}\text { Psychiatrist or addiction medicine specialist billing in practice where } \\
\text { patient received Most AUD care }\end{array}$} \\
\hline Yes & 1.01 & $0.99,1.02$ \\
\hline No & Reference & - \\
\hline \multicolumn{3}{|c|}{ Moderate or severe AUD diagnosis at baseline } \\
\hline Yes & 1.08 & $1.06,1.09$ \\
\hline No & Reference & - \\
\hline \multicolumn{3}{|c|}{ OUD diagnosis at baseline } \\
\hline Yes & 1.10 & $1.08,1.13$ \\
\hline No & Reference & - \\
\hline \multicolumn{3}{|c|}{ SUD diagnosis besides AUD or OUD at baseline } \\
\hline Yes & 1.05 & $1.03,1.07$ \\
\hline No & Reference & - \\
\hline \multicolumn{3}{|c|}{ Acute alcohol-related medical diagnosis at baseline } \\
\hline Yes & 1.09 & $1.06,1.11$ \\
\hline No & Reference & - \\
\hline \multicolumn{3}{|c|}{ Chronic alcohol-related medical diagnosis at baseline } \\
\hline Yes & 1.04 & $1.02,1.06$ \\
\hline No & Reference & - \\
\hline \multicolumn{3}{|c|}{ Severe mental illness diagnosis at baseline } \\
\hline Yes & 1.08 & $1.06,1.10$ \\
\hline No & Reference & - \\
\hline \multicolumn{3}{|c|}{ Index AUD visit setting } \\
\hline Inpatient/ED & 1.14 & $1.12,1.15$ \\
\hline Intermediate & 1.04 & $1.02,1.07$ \\
\hline Outpatient & Reference & - \\
\hline \multicolumn{3}{|c|}{ Year of index AUD visit } \\
\hline 2018 & 0.71 & $0.68,0.74$ \\
\hline 2017 & 0.90 & $0.87,0.92$ \\
\hline 2016 & 0.93 & $0.91,0.96$ \\
\hline 2015 & 0.94 & $0.92,0.97$ \\
\hline 2014 & 0.96 & $0.93,0.98$ \\
\hline 2013 & 0.90 & $0.88,0.92$ \\
\hline 2012 & 0.84 & $0.82,0.86$ \\
\hline 2011 & 0.85 & $0.83,0.87$ \\
\hline 2010 & 0.92 & $0.90,0.94$ \\
\hline 2009 & 0.99 & $0.97,1.02$ \\
\hline 2008 & Reference & - \\
\hline
\end{tabular}

Notes: We estimated the risk of benefit loss controlling for patient characteristics and whether the practice where the patient received the majority of AUD care included a psychiatrist or addition medicine specialist, accounting for the competing risk of AUD medication use

2019). In addition to the CDC list, we added Korsakoff's dementia to the list of chronic alcohol related disorders.
Table 7 Comparison of Individuals with an Index AUD Visit Who Were Dropped Due to Missing Data Versus Those Not Dropped Due to Missing Data (and Thus in Study Cohort)

\begin{tabular}{llll}
\hline \hline & $\begin{array}{l}\text { Individuals } \\
\text { dropped due to } \\
\text { missing data }(\boldsymbol{n}= \\
\mathbf{3 3 1 5})\end{array}$ & $\begin{array}{l}\text { Individuals in } \\
\text { study cohort } \\
(\boldsymbol{n}=\mathbf{1 5 1 , 1 2 8})\end{array}$ & $\begin{array}{l}\text { Chi-square } \\
\text { probability }\end{array}$ \\
\hline Age & & & \\
$17-25$ & $21.7 \%$ & $17.7 \%$ & $<0.001$ \\
$26-35$ & $18.6 \%$ & $18.9 \%$ & \\
$36-45$ & $21.0 \%$ & $22.8 \%$ & \\
$46-55$ & $22.8 \%$ & $25.7 \%$ & \\
56 and & $15.8 \%$ & $14.9 \%$ & \\
over & & & \\
Employee status & & \\
Employee & $64.0 \%$ & $63.8 \%$ & \\
Dependent & $36.0 \%$ & $36.2 \%$ & \\
Baseline moderate or severe AUD diagnosis & \\
Yes & $57.7 \%$ & $61.7 \%$ & \\
No & $42.3 \%$ & $38.3 \%$ & \\
\hline
\end{tabular}

\section{Description of Competing Risk Model}

Loss of benefits precludes observing a medication fill and may be informative for a future medication fill. For instance, if a subject dropped health coverage because they could not afford the co-payment for the medication prior to medication fill, this information suggests that they will never fill the drug and so it is informative. We thus treated loss of benefits as a competing risk. We estimated two cause-specific hazard functions, one for first AUD medication fill and one for loss of benefits. Our main interest is in the hazard function for first AUD medication which describes the rate of occurrence of a first AUD medication fill among all subjects who are currently enrolled and who have not yet filled an AUD medication. We assumed Cox proportional hazards models for each cause and report hazard ratios and corresponding $95 \%$ confidence intervals. We assessed the proportional hazards assumption by examining the Schoenfeld residuals over time (Schoenfeld D., Biometrika 1982); we plotted the deviance residuals against the linear predictor, the estimated log risk, to identify any systematic patterns. Competing risk models were estimated in SAS using the PHREG procedure.

Results: Proportionality and Residuals

We did not find evidence to contradict our proportional hazards assumption based on the Schoenfeld residuals. When examining the deviance residuals, all residuals were between 3 and -3 for both the loss of benefit events and treatment events. For both event types, the model tended to underpredict events for males and employees

Factors Associated with Likelihood of Losing Benefits before an AUD Medication Fill. Factors associated with a greater likelihood of losing benefits before an AUD medication fill are younger age (relative to age 56 and above), employee status (vs. dependent), residence in the Midwest (relative to the South), moderate or severe AUD 
Table 8 Results from Competing Risk Model of Alcohol Use Disorder (AUD) Medication Use after Index AUD Visit, Accounting for Risks of Benefit Loss-Excludes Individuals with Baseline Diagnoses of Either OUD Or seizure disorders

\begin{tabular}{|c|c|c|}
\hline & Hazard ratio & $95 \%$ confidence interval \\
\hline \multicolumn{3}{|l|}{ Age } \\
\hline $17-25$ & 0.65 & $0.61,0.70$ \\
\hline $26-35$ & 1.23 & $1.17,1.30$ \\
\hline $36-45$ & 1.46 & $1.39,1.53$ \\
\hline $46-55$ & 1.29 & $1.23,1.36$ \\
\hline 56 and over & Reference & - \\
\hline \multicolumn{3}{|l|}{ Male } \\
\hline Yes & 0.70 & $0.68,0.73$ \\
\hline No & Reference & - \\
\hline \multicolumn{3}{|l|}{ Employee status } \\
\hline Employee & 0.89 & $0.86,0.92$ \\
\hline Dependent & Reference & - \\
\hline \multicolumn{3}{|l|}{ Rural } \\
\hline Yes & 0.97 & $0.91,1.04$ \\
\hline No & Reference & - \\
\hline \multicolumn{3}{|l|}{ Region } \\
\hline Northeast & 0.77 & $0.74,0.80$ \\
\hline Midwest & 0.85 & $0.81,0.89$ \\
\hline West & 0.91 & $0.87,0.94$ \\
\hline South & Reference & - \\
\hline \multicolumn{3}{|c|}{ Median household income for zip code of residence } \\
\hline Quartile 1 & Reference & - \\
\hline Quartile 2 & 1.16 & $1.11,1.22$ \\
\hline Quartile 3 & 1.30 & $1.24,1.36$ \\
\hline Quartile 4 & 1.54 & $1.47,1.61$ \\
\hline \multicolumn{3}{|c|}{ Psychiatrist or addiction medicine specialist billing in practice where patient received most AUD care } \\
\hline Yes & 1.15 & $1.12,1.19$ \\
\hline No & Reference & - \\
\hline \multicolumn{3}{|c|}{ Moderate or severe AUD diagnosis at baseline } \\
\hline Yes & 2.06 & $1.99,2.14$ \\
\hline No & Reference & - \\
\hline \multicolumn{3}{|c|}{ SUD diagnosis besides AUD or OUD at baseline } \\
\hline Yes & 0.85 & $0.81,0.89$ \\
\hline No & Reference & - \\
\hline \multicolumn{3}{|c|}{ Acute alcohol-related medical diagnosis at baseline } \\
\hline Yes & 0.75 & $0.71,0.80$ \\
\hline No & Reference & - \\
\hline \multicolumn{3}{|c|}{ Chronic alcohol-related medical diagnosis at baseline } \\
\hline Yes & 1.10 & $1.05,1.14$ \\
\hline No & Reference & - \\
\hline \multicolumn{3}{|c|}{ Severe mental illness diagnosis at baseline } \\
\hline Yes & 1.32 & $1.28,1.37$ \\
\hline No & Reference & - \\
\hline \multicolumn{3}{|c|}{ Index AUD visit setting } \\
\hline Inpatient/ED & 1.27 & $1.23,1.31$ \\
\hline Intermediate & 1.11 & $1.05,1.19$ \\
\hline Outpatient & Reference & - \\
\hline \multicolumn{3}{|c|}{ Year of index AUD visit } \\
\hline 2018 & 1.98 & $1.85,2.12$ \\
\hline 2017 & 1.79 & $1.67,1.91$ \\
\hline 2016 & 1.62 & $1.52,1.74$ \\
\hline 2015 & 1.52 & $1.42,1.63$ \\
\hline 2014 & 1.46 & 1.361 .56 \\
\hline 2013 & 1.36 & $1.26,1.46$ \\
\hline 2012 & 1.27 & $1.18,1.36$ \\
\hline 2011 & 1.29 & $1.20,1.38$ \\
\hline 2010 & 1.23 & $1.15,1.32$ \\
\hline 2009 & 1.13 & $1.06,1.21$ \\
\hline 2008 & Reference & - \\
\hline
\end{tabular}

Notes: We estimated the risk of AUD medication use controlling for patient characteristics and whether the practice where the patient received the majority of AUD care included a psychiatrist or addiction medicine specialist, accounting for the competing risk of loss of benefits. The baseline period was 90 days before and including the index AUD visit

diagnosis at baseline, co-occurring opioid use disorder diagnosis at baseline, co-occurring SUD diagnosis other than AUD or OUD at baseline, severe mental illness diagnosis at baseline, acute alcohol-related medical diagnosis at baseline, and having an index AUD visit that occurred in an inpatient/ED setting or in an intermediate setting (relative to outpatient setting) (Appendix Table 6). Rural residence, higher median household income, and later year of index AUD visit were associated with a lower likelihood of losing benefits before a medication fill. 